\title{
FORMULAS FOR THE NUMBER OF BINOMIAL COEFFICIENTS DIVISIBLE BY A FIXED POWER OF A PRIME
}

\author{
F. T. HOWARD
}

ABstract. Define $\theta_{j}(n)$ as the number of binomial coefficients $\left(\begin{array}{l}n \\ s\end{array}\right)$ divisible by exactly $p^{j}$. A formula for $\theta_{2}(n)$ is found, for all $n$, and formulas for $\theta_{j}(n)$ for $n=a p^{k}+b p^{r}$ and $n=c_{1} p^{k_{1}}+\cdots+c_{m} p^{k_{m}}$ $\left(k_{1} \geqq j, k_{i+1}-k_{i} \geqq j\right.$ for $\left.i=1, \cdots, m-1\right)$ are derived.

1. Introduction. Let $p$ be a fixed prime and let $\theta_{j}(n)$ denote the number of binomial coefficients $\left(\begin{array}{l}n \\ s\end{array}\right)(s=0,1, \cdots, n)$ divisible by exactly $p^{j}$.

If we put

$$
n=c_{0}+c_{1} p+\cdots+c_{r} p^{r} \quad\left(0 \leqq c_{i}<p\right)
$$

it is well known [3] that

$$
\theta_{0}(n)=\left(c_{0}+1\right)\left(c_{1}+1\right) \cdots\left(c_{r}+1\right) .
$$

The evaluation of $\theta_{j}(n)$ for arbitrary $j$ appears to be more difficult, however. Carlitz [1] has proved that

$\theta_{1}(n)=\sum_{k=0}^{r-1}\left(c_{0}+1\right) \cdots\left(c_{k-1}+1\right)\left(p-c_{k}-1\right) c_{k+1}\left(c_{k+2}+1\right) \cdots\left(c_{r}+1\right)$

and he has found formulas for $\theta_{j}(n)$ for the following values of $n$ :

$$
\begin{array}{ll}
a p^{r}+b p^{r+1} & (0 \leqq a<p, 0 \leqq b<p), \\
b+a p+a p^{2}+\cdots+a p^{r+j} & (0<a<p, b=a \text { or } a-1) .
\end{array}
$$

The writer [4] has considered this problem for $p=2$ and has found formulas for $\theta_{j}(n), 1 \leqq j \leqq 4$, and for arbitrary $j$ has evaluated $\theta_{j}(n)$ for a number of special values of $n$. These formulas are valid only for $p=2$, however.

In this paper we find formulas for $\theta_{2}(n)$ for all $n$ and for $\theta_{j}(n)$ for the following values of $n$ :

$$
\begin{array}{ll}
a p^{k}+b p^{r} & (0<a<p, 0<b<p, k<r), \\
c_{1} p^{k_{1}}+\cdots+c_{m} p^{k_{m}} & \left(0<c_{i}<p, j \leqq k_{1}, j<k_{i+1}-k_{i}\right) .
\end{array}
$$

Received by the editors August 2, 1971.

AMS (MOS) subject classifications (1970). Primary 05A10, 05A15.

Key words and phrases. Binomial coefficient, prime number. 
We shall use the following rule, which was proved by Kummer [2, p. 70]. Put

$$
\begin{array}{lrr}
\text { (1.2) } & s=a_{0}+a_{1} p+\cdots+a_{r} p^{r} & \left(0 \leqq a_{i}<p\right), \\
(1.3) & n-s=b_{0}+b_{1} p+\cdots+b_{r} p^{r} & \left(0 \leqq b_{i}<p\right), \\
a_{0}+b_{0}=c_{0}+\varepsilon_{0} p, \varepsilon_{0}+a_{1}+b_{1}=c_{1}+\varepsilon_{1} p, & \cdots, \\
& \varepsilon_{r-1}+a_{r}+b_{r}=c_{r}+\varepsilon_{r} p,
\end{array}
$$

where each $\varepsilon_{i}=0$ or 1 . Let $N$ be the exponent of the highest power of $p$ that divides $\left(\begin{array}{l}n \\ s\end{array}\right)$. Then we have $N=\varepsilon_{0}+\varepsilon_{1}+\cdots+\varepsilon_{r}$.

2. Evaluation of $\theta_{2}(n)$. If $n$ is given by (1.1) and $s$ and $n-s$ are given by (1.2) and (1.3), it is clear that $N=2$ if and only if exactly two of the $\varepsilon$ 's are equal to 1 , and $\varepsilon_{r}=0$. There are two possibilities. Either $\varepsilon_{k}=$ $\varepsilon_{k+1}=1$ for some $0 \leqq k \leqq r-2$ and all other $\varepsilon$ 's $=0$ or $\varepsilon_{k}=1, \varepsilon_{m}=1$ for some $0 \leqq k \leqq r-3, k+2 \leqq m \leqq r-1$ and all other $\varepsilon$ 's $=0$. In the first case we can take

$$
\begin{aligned}
a_{k} & =c_{k}+1, \cdots, p-1 ; \quad a_{k+1}=c_{k+1}, \cdots, p-1 ; \\
a_{k+2} & =0, \cdots, c_{k+2}-1 .
\end{aligned}
$$

So we have $\left(p-c_{k}-1\right)\left(p-c_{k+1}\right) c_{k+2}$ choices and the remaining $a$ 's can be chosen in $A_{k}$ ways, where

$$
A_{k}=\left[\prod_{i=0}^{r}\left(c_{i}+1\right)\right] /\left[\left(c_{k}+1\right)\left(c_{k+1}+1\right)\left(c_{k+2}+1\right)\right] .
$$

In the second case, we can take

$$
\begin{aligned}
a_{k} & =c_{k}+1, \cdots, p-1 ; & a_{k+1} & =0, \cdots, c_{k+1}-1, \\
a_{m} & =c_{m}+1, \cdots, p-1 ; & a_{m+1} & =0, \cdots, c_{m+1}-1,
\end{aligned}
$$

so there are $\left(p-c_{k}-1\right) c_{k+1}\left(p-c_{m}-1\right) c_{m+1}$ choices. The remaining $a$ 's can be selected in $B_{k, m}$ ways, where

$$
B_{k, m}=\left[\prod_{i=0}^{r}\left(c_{i}+1\right)\right] /\left[\left(c_{k}+1\right)\left(c_{k+1}+1\right)\left(c_{m}+1\right)\left(c_{m+1}+1\right)\right]
$$

Thus we have

$$
\begin{aligned}
\theta_{2}(n)= & \sum_{k=0}^{r-2}\left(p-c_{k}-1\right)\left(p-c_{k+1}\right) c_{k+2} A_{k} \\
& +\sum_{m=k+2}^{r-1} \sum_{k=0}^{r-3}\left(p-c_{k}-1\right) c_{k+1}\left(p-c_{m}-1\right) c_{m+1} B_{k, m},
\end{aligned}
$$

where $A_{k}$ and $B_{k, m}$ are defined by (2.1) and (2.2) respectively. 
For example,

$$
\begin{aligned}
\theta_{2}\left(a+b p+c p^{2}\right)= & (p-a-1)(p-b) c \\
\theta_{2}\left(a+b p+c p^{2}+d p^{3}\right)= & (p-a-1)(p-b) c(d+1) \\
& +(a+1)(p-b-1)(p-c) d \\
& +(p-a-1) b(p-c-1) d .
\end{aligned}
$$

This method does not appear to be very practical for evaluating $\theta_{j}(n)$ for $j>2$.

3. Special evaluations. We can use Kummer's theorem to evaluate $\theta_{j}\left(a p^{k}+b p^{r}\right)$, where $r>k, 0<a<p, 0<b<p$. Suppose $k \geqq j$ and $r-k>j$. Then there are three ways to have exactly $j$ of the $\varepsilon$ 's equal to 1 :

(1) $\varepsilon_{r-j}=\varepsilon_{r-j+1}=\cdots=\varepsilon_{r-1}=1$, all other $\varepsilon$ 's $=0$;

(2) $\varepsilon_{k-j}=\varepsilon_{k-j+1}=\cdots=\varepsilon_{k-1}=1$, all other $\varepsilon$ 's $=0$;

(3) $\varepsilon_{k-m}=\varepsilon_{k-m+1}=\cdots=\varepsilon_{k-1}=1, \varepsilon_{r-h}=\cdots=\varepsilon_{r-1}=1,1 \leqq m \leqq j-1, h=$ $j-m$, all other $\varepsilon$ 's $=0$.

If $s$ is given by (1.2), in the first case we can take

$$
\begin{aligned}
a_{r-j} & =1, \cdots, p-1 ; \quad a_{i}=0, \cdots, p-1 \quad(r-j+1 \leqq i \leqq r-1) ; \\
a_{r} & =0, \cdots, b-1 ; \quad a_{k}=0, \cdots, a,
\end{aligned}
$$

so there are $(p-1) p^{j-1}(a+1) b$ choices. Using similar reasoning in the other two cases, we have

$$
\begin{aligned}
\theta_{j}\left(a p^{k}+b p^{r}\right)= & (p-1) p^{j-1}(a+1) b \\
& +(p-1) p^{j-1} a(b+1)+(j-1)(p-1)^{2} p^{j-2} a b \\
& (k \geqq j, r>k+j) .
\end{aligned}
$$

Similarly we have

$$
\begin{aligned}
\theta_{j}\left(a p^{k}+b p^{r}\right) & \\
= & (p-1) p^{j-1}(a+1) b+k(p-1)^{2} p^{j-2} a b \quad(k<j, r>k+j), \\
= & (p-a-1) p^{j-1} b+k(p-1)^{2} p^{j-2} a b \quad(k<j, r=k+j), \\
= & (p-1) p^{j-1} a(b+1)+(p-1) p^{j-2}(p-a) b \\
& \quad+(r-k-1)(p-1)^{2} p^{j-2} a b \quad(k \geqq j, r<k+j), \\
= & (p-1) p^{j-1} a(b+1)+(p-a-1) p^{j-1} b \\
& +(j-1)(p-1)^{2} p^{j-2} a b \quad(k \geqq j, r+k=j), \\
= & (p-1) p^{j-2}(p-a) b+(r-j)(p-1)^{2} p^{j-2} a b
\end{aligned}
$$

We next evaluate $\theta_{j}(n)$ for $(k<j, r<k+j, r \geqq j)$.

$$
n=c_{1} p^{k_{1}}+c_{2} p^{k_{2}}+\cdots+c_{m} p^{k_{m}} \quad\left(k_{1} \geqq j, k_{i+1}-k_{i}>j\right) .
$$


Using Kummer's theorem, we need to determine the number of ways we can have exactly $j$ of the $\varepsilon$ 's equal to 1 . Let $1 \leqq u \leqq m$ and choose $u$ of the $c_{i}$ 's. Call them $c_{i_{1}}, \cdots, c_{i_{u}}$. Assign to each $c_{i_{w}}$ a number $t_{w}, 1 \leqq t_{w}$, such that $t_{1}+t_{2}+\cdots+t_{u}=j$. This can be done in $\left(\begin{array}{l}j-1 \\ u-1\end{array}\right)$ ways, since there are $\left(\begin{array}{l}j-1 \\ u-1\end{array}\right)$ different ways of distributing $j$ nondistinct objects into $u$ distinct cells with no cell left empty. We wish to have $\varepsilon_{v}=1 \quad\left(v=i_{w}-h, 1 \leqq h \leqq t_{w}\right.$, $1 \leqq w \leqq u$ ) and all other $\varepsilon$ 's equal to 0 . If $s$ is given by (1.2) we can take

$$
\begin{aligned}
a_{v} & =1, \cdots, p-1 & & \left(v=i_{w}-t_{w}, 1 \leqq w \leqq u\right), \\
& =0, \cdots, p-1 & & \left(v=i_{w}-h, 1 \leqq h \leqq t_{w}-1,1 \leqq w \leqq u\right), \\
& =0, \cdots, c_{v}-1 & & \left(v=i_{w}, 1 \leqq w \leqq u\right) .
\end{aligned}
$$

Thus for a given $u$ and a given selection $i_{1}, \cdots, i_{u}$, there are $\left(\begin{array}{l}j-1 \\ u-1\end{array}\right)(p-1)^{u} p^{j-u} c_{i_{1}} \cdots c_{i_{u}}\left(c_{1}+1\right) \cdots\left(c_{m}+1\right) /\left(c_{i_{1}}+1\right) \cdots\left(c_{i_{u}}+1\right)$ different ways to have $j$ of the $\varepsilon$ 's equal to 1 . Therefore

$$
\theta_{j}(n)=\sum_{u=1}^{m}\left(\begin{array}{l}
j-1 \\
u-1
\end{array}\right)(p-1)^{u} p^{j-u} E_{u}
$$

where $n$ is given by (3.7) and

$$
E_{u}=\sum c_{i_{1}} \cdots c_{i_{u}}\left(c_{1}+1\right) \cdots\left(c_{m}+1\right) /\left[\left(c_{i_{1}}+1\right) \cdots\left(c_{i_{u}}+1\right)\right],
$$

the sum being over all subsets $\left\{i_{1}, \cdots, i_{u}\right\}$ of $\{1, \cdots, m\}$ such that $i_{1}<i_{2}<\cdots<i_{u}$.

For example, if $n=a p^{k_{1}}+b p^{k_{2}}+c p^{k_{3}}, k_{1} \geqq j, k_{2}-k_{1}>j, k_{3}-k_{2}>j$, then

$$
\begin{aligned}
\theta_{j}(n)= & (p-1) p^{j-1}[a(b+1)(c+1)+(a+1) b(c+1)+(a+1)(b+1) c] \\
& +(j-1)(p-1)^{2} p^{j-2}[a b(c+1)+a(b+1) c+(a+1) b c] \\
& +\left(\begin{array}{c}
j-1 \\
2
\end{array}\right)(p-1)^{3} p^{j-3} a b c .
\end{aligned}
$$

If $n$ is given by (3.7) and $c_{1}=c_{2}=\cdots=c_{k} \equiv a$, then (3.8) becomes

$$
\theta_{j}(n)=\sum_{u=1}^{m}\left(\begin{array}{l}
j-1 \\
u-1
\end{array}\right)\left(\begin{array}{l}
m \\
u
\end{array}\right)(p-1)^{u} p^{j-u}(a+1)^{m-u} a^{u} .
$$

If $n$ is given by (3.7), except that $k_{1}=j-1$ and $k_{i+1}-k_{i}=j$, we have, by an argument similar to the one above,

$$
\theta_{j}(n)=p^{j-1} F+\sum_{u=2}^{m}\left(\begin{array}{l}
j-1 \\
u-1
\end{array}\right)(p-1)^{u} p^{j-u} E_{u},
$$


where $E_{u}$ is defined by (3.9) and

$$
F=\sum_{i=1}^{m-1}\left(p-c_{i}-1\right) c_{i+1}\left(c_{1}+1\right) \cdots\left(c_{m}+1\right) /\left[\left(c_{i}+1\right)\left(c_{i+1}+1\right)\right] .
$$

If $k_{1} \geqq j$ and $k_{i+1}-k_{i}=j$, then

$$
\begin{aligned}
\theta_{j}(n)= & (p-1) p^{j-1} c_{1}\left(c_{2}+1\right) \cdots\left(c_{m}+1\right) \\
& +p^{j-1} F+\sum_{u=2}^{m}\left(\begin{array}{l}
j-1 \\
u-1
\end{array}\right)(p-1)^{u} p^{j-u} E_{u} .
\end{aligned}
$$

If $k_{1}=0, k_{i+1}-k_{i}>j$, then

$$
\theta_{j}(n)=\sum_{u=1}^{m}\left(\begin{array}{l}
j-1 \\
u-1
\end{array}\right)(p-1)^{u} p^{j-u} G_{u}
$$

where

$$
G_{u}=\sum c_{i_{1}} \cdots c_{i_{u}}\left(c_{1}+1\right) \cdots\left(c_{m}+1\right) /\left[\left(c_{i_{1}}+1\right) \cdots\left(c_{i_{u}}+1\right)\right],
$$

the sum being over all subsets $\left\{i_{1}, \cdots, i_{u}\right\}$ of $\{2, \cdots, m\}$ such that $i_{1}<i_{2}<\cdots<i_{u}$.

If $n$ is given by (3.7) and $c_{1}=c_{2}=\cdots=c_{m}=a$, then (3.11) becomes

$$
\begin{aligned}
\theta_{j}(n)= & (m-1) a(p-a-1) p^{j-1}(a+1)^{m-2} \\
& +\sum_{u=2}^{m}\left(\begin{array}{l}
j-1 \\
u-1
\end{array}\right)\left(\begin{array}{l}
m \\
u
\end{array}\right)(p-1)^{u} p^{j-u}(a+1)^{m-u} a^{u} ;
\end{aligned}
$$

(3.12) becomes

(3.13) becomes

$$
\begin{aligned}
\theta_{j}(n)= & (p-1) p^{j-1} a(a+1)^{m-1} \\
& +(m-1)(p-a-1) a(a+1)^{m-2} p^{j-1} \\
& +\sum_{u=2}^{m}\left(\begin{array}{l}
j-1 \\
u-1
\end{array}\right)\left(\begin{array}{l}
m \\
u
\end{array}\right)(p-1)^{u} p^{j-u}(a+1)^{m-u} a^{u}
\end{aligned}
$$

$$
\theta_{j}(n)=\sum_{u=1}^{m-1}\left(\begin{array}{l}
j-1 \\
u-1
\end{array}\right)\left(\begin{array}{c}
m-1 \\
u
\end{array}\right)(p-1)^{u} p^{j-u}(a+1)^{m-u} a^{u}
$$

\section{REFERENCES}

1. L. Carlitz, The number of binomial coefficients divisible by a fixed power of a prime, Rend. Circ. Mat. Palermo (2) 16 (1967), 299-320. MR 40 \#2554.

2. L. E. Dickson, History of the theory of numbers. Vol. 1, Publication no. 256, Carnegie Institution of Washington, Washington, D.C., 1919.

3. N. J. Fine, Binomial coefficients modulo a prime, Amer. Math. Monthly 54 (1947), 589-592. MR 9, 331.

4. F. T. Howard, The number of binomial coefficients divisible by a fixed power of 2, Proc. Amer. Math. Soc. 29 (1971), 236-242.

Department of Mathematics, Wake Forest University, Winston-Salem, North CAROLINA 27109 